\title{
Enhanced Energy Harvesting for IOT based Fuzzy Logics by using Gaussian Membership Functions
}

\author{
Harmanjot Singh \\ Associate Professional: Product Developer \\ DXC Technology, Chennai
}

\author{
Chanpreet Kaur \\ Guru Nanak Dev University, Amritsar
}

\begin{abstract}
Energy harvesting is a capable approach for the developing IOT. Thus, nodes in IOTs are battery-powered, thus a lowpower feature is a fundamental requirement. For batterypowered nodes, methods are required in order to reduce possible energy consumption. This report delivers that numerous high efficiency techniques are suggested until now for improved power consumption. Beyond these individuals hairy based IOTs has proved very efficient success but still it can be enhanced additional by simply introducing additional membership rights functions. The reason for this kind of report will be to propose Gaussian sensible models based membership rights function for optimizing in addition to decreasing utilization sleep mode.
\end{abstract}

\section{Keywords}

Internet of Things (IOT), Wireless Energy Harvesting Units, Fuzzy Logic and Gaussian Membership Functions.

\section{INTRODUCTION}

The Internet of Things commonly named as IOT, is an important subject in the field of advance industry and research, approach, as well as structuring circles and the wellknown media. This advance is exemplified in a broad variety of given items, sensors, frameworks that use progressions in figuring power, hardware scaling down, as well as organize the interconnections between the devices to provide new capacities not beforehand possible. While the expression "Web of Things" is moderately new, the thought of joining systems, Pc's to the screen and control the various electronic gadget's been around for a large amount of time.

The IOT twists around extended machine-to-machine communication; it depends on distributed computing and systems of information social occasion sensors; it's versatile, virtual, and quick affiliation. The Internet of Things really gets alongside the association of sensors and machines. That's to state, the honest to goodness esteem that the Internet of Things makes are at the crossing point of gathering information and using it. Cloud-based applications are the very best way of using utilized information.

\subsection{Wireless Energy harvesting Unit}

The WEH unit receives the transferred radio waves utilizing an antenna as well transfers the conventional RF energy into a mild direct current (DC) source of energy to offer the sensor device. Usually, poor IOT, wireless sensor networks as well as radio-frequency identification (RFID) tags; wireless powers are frequently divided into various classes [6]:

1) Dedicated source: These sources are deployed start using a conventional energy provide about the device. This is often optimized regarding frequency as well as highest capacity that is eligible the sensor devices. Mobile node is a particular DS.

2) Ambient source: These kinds of source are further placed in two types:

a) Static or anticipated ambient sources, that have been transmitters which radiate stable control of their time, aren't optimized (such as, regarding frequency as well as transmitted power) toward offer the sensor device. Sink base station, broadcast radio as well as TV are degrees of predictable ambient sources.

b) Dynamic or unknown ambient sources that have been transmitters that broadcast periodically in such a way not controlled over the IOT scheme. Harvesting energy for example sources need WEH to keep an eye on the channel for harvesting opportunities. Wi-Fi access points, microwave radio links are degrees of unknown ambient sources.

\section{PROPOSED ALGORITHM}

\subsection{Fuzzy Logic:}

Fuzzy approach will depend on simple rules, that are super easy to use and take less time. Use of this tactic will work for pixel level image fusion. This plan forms an alternative solution replacement for many conventional approaches that is with some other host of empirical relations. Empirical approaches are time intensive and make up a low association. Fuzzy Logic method may also become sensor fusion. This sensor fusion could are persons in a variety of sensor fusion if you do the functions may be input and decision may be output. The laptop can often be skilled to the input information on the sensors. The essence is usually to correlate the given sensory inputs with some decision outputs. After developing computer another input data works to learn the performance together with the system.

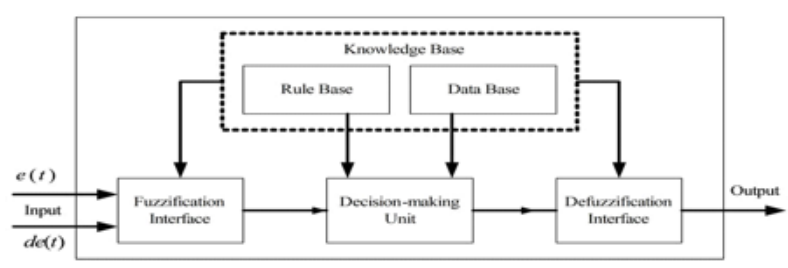

Fig 1: Fuzzy logic

\subsubsection{Fuzzy membership function}

Fuzzy categorization is the mainly applying fuzzy logic which utilized to take care of classification problems. In continuing enlargement of a fuzzy classification scheme, the top should be to make membership functions as well as to get many appropriate fuzzy rules inside the fuzzy classification scheme [5]. Fuzzy membership functions could be formulated specialized in expert knowledge approach alongside alternative are use data driven approach. These approaches 
relate with the concept to automatically or manually by means of machine learning process specialized in training instances respectively, commonly expert knowledge is applied to formulate membership function range in the event then rules for inference. It has an advantage that it connect to domain knowledge however it could be very subjective with different experts to generate different membership rules and functions for the same application. Additional approach of generation of fuzzy membership functions on such basis while the input information will transfer information into semantic terms.

Fuzzy logic idea can be compared to the human beings being's feeling and inference process. Unlike classical control strategy, which is a real point-to-point control, fuzzy logic control can be a range-to-point or range-to-range control [6]. The production of a fuzzy controller emanates from fuzzifications of both inputs and outputs when using the associated membership functions. A crisp input may be become different folks the associated membership functions predicated on its value. By using perspective, the production of a fuzzy logic controller is founded on its memberships of various membership functions that is regarded as a range of inputs.

To implement fuzzy logic process to a true application necessitates following three steps:

1. Fuzzifications- change over traditional information or fresh information into fluffy information or Membership Functions

2. Fluffy Inference Process - join enrollment capacities with the control standards to determine the fluffy yield 3 .

3. Defuzzification - use diverse strategies to figure each related yield and place them into a table: the lookup table. Get the yield from the lookup table in view of the present contribution within an application

\section{a) Gaussian Membership Function:}

Gaussian fuzzy membership functions are well liked inside the fuzzy logic, because they are the outcomes of fuzzy systems and radial basis function (RBF) neural networks. Fuzzy membership function that can often be accustomed to represent vague, semantic terms could be the Gaussian and that is written by:

$$
\mu A i(x)=\exp (-(c i-x) 22 \sigma 2 i)(1)
$$

where ci and $\sigma i$ are the centre as well as width of the ith fuzzy set $\mathrm{Ai}$, respectively.

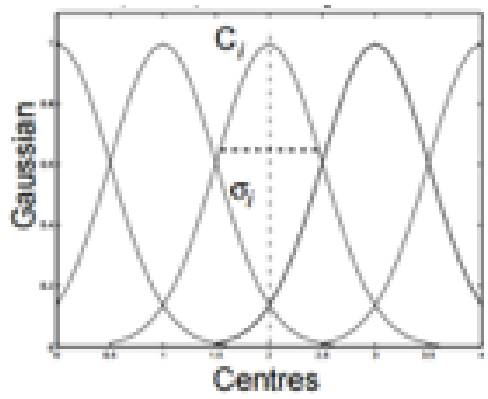

Fig 2: Representing Gaussian functions

\section{RELATED WORK}

Collata et al. (2015) [2] proposed the energy proficient is the principle necessity generates wireless protocol to utilized in IOT. BLE has a potential in turning into a critical technological improvement with the IOT within small electrical power, little attempt, along with tiny devices. Mario.collotta (2014) [3] proposed architecture, comes with a strategy to power the sensor nodes based upon piezoelectric materials which let it produce potential energy making the most of the vibration produced with the passage of vehicles about the road. Toklu et al. (2014) [4] presented the energy consumption is the major issue as the devices, processors and memory forming sensor networks are small and usually battery powered. Eleonora Borgia et al. (2014) [5] discussed that the Internet of Things (IoT) is a new paradigm that merges aspects and technologies coming from different approaches. F. Zarafshan (2012) [6] work with a fuzzy logic controller to relieve the communication overheads during flooding and routing your data stream through the source(s) to some sink node. The fuzzy logic controller evaluates the potentiality of intermediate nodes to coordinate in mission, dependant on each node's traffic load, energy residual and size of information that is maintained. Rani et al. (2012) [7] considered in the paper incorporate boosting network.

\section{GAPS IN LITERATURE}

Kamalinejad, Pouya, et al. [1] presented a summary of technologies for proficient WEH, focus the time of WEHenabled IOT strategy, as well as briefly review the long term trends. By conducting the review, it can be obtained that the earlier study has neglected many issues that might enhance the performance of IOTs further.

1. The existing literature has not considered Gaussian functional shapes as membership functions of fuzzy logic that could enhance the accuracy as well as strength of IOTs.

2. The existing work has not considered the outcome of overheads that might be degrading the computational performance of IOTs.

3. Effects of the node harvesting is also ignored in the majority of existing fuzzy based energy efficient IOTs.

\section{METHODOLOGY}

\subsection{Proposed Algorithm}

STEP 1: Randomly initialize the IOT nodes with their respective membership values using the equation as follows:

$\sum_{\mathrm{j}=1}^{c} \mu \mathrm{k}\left(\mathrm{x}_{\mathrm{a}}\right)=1 \mathrm{a}=1,2 \ldots \mathrm{L}$

STEP 2: Calculate the centroid for IOT nodes using following formula,

$$
\frac{\left.\dot{C}_{j}=\sum_{k=1}^{c} \mu j\left(x_{a}\right)\right]^{n} x_{i}}{\left.\dot{C}_{j}=\sum_{k=1}^{c} \mu j\left(x_{a}\right)\right]^{n}}
$$

STEP 3: Calculate dissimilarity between the IOT nodes and centroid using the Euclidean distance as given by Eq. (3)

$D_{i}=\sqrt{\left(a_{2}-a_{1}\right)^{2}+\left(b_{2}-b_{1}\right)^{2}}$

STEP 4: Update the new membership matrix using the equation for all IOT nodes, 
$\mu_{\mathrm{j}}\left(\mathrm{i}_{\mathrm{a}}\right)=\frac{\left[\frac{1}{\mathrm{~d}_{\mathrm{ja}}}\right]^{1 / \mathrm{m}-1}}{\sum_{\mathrm{L}=1}^{\dot{C}}\left[\frac{1}{\mathrm{~d}_{\mathrm{Ka}}}\right]^{1 / \mathrm{m}-1}}$

Here $m$ is a fuzzifications parameter

The range $m$ is always positive.

STEP 5: Go back to step 2, unless the centroid are not changing.

STEP 6: Initialize communication phase between user and controlling node $(\mathrm{CN})$

(1) Compute initial probe distribution

(2) Compute initial fitness matrix.

(3) Assign initial probe acceleration

STEP 7: Multicast data from controlling node $(\mathrm{CN})$ to IoT nodes

For $\mathrm{j}=1$ to $\mathbf{N}_{\mathrm{t}}$

(b) Compute probe position vector for each IoT $\overrightarrow{\mathrm{R}}_{\mathrm{J}, 1}^{\mathrm{P}} 1 \leq \mathrm{P} \leq \mathrm{N}_{\mathrm{P}}$

(c) Retrieve errant probes $(1 \leq \mathrm{p} \mathrm{N}$ p); If

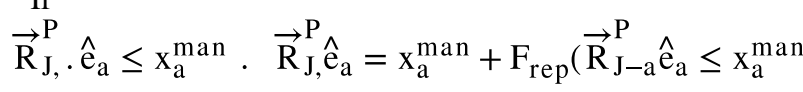

If

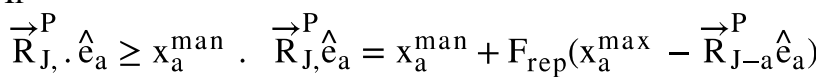

(d) Compute communication matrix for current probe distribution.

(e) Compute accelerations using current probe distribution

(f) Increment $\mathrm{F}_{\text {rep by }} \Delta \mathrm{F}_{\text {rep }}$ : if $\mathrm{F}_{\text {rep }}=\Delta \mathrm{F}_{\text {rep }}$.

(g) If j MOD 20-0. Shrink $\Omega$ around $\overrightarrow{\mathrm{R}}_{\text {BEST }}$

STEP 8: For each candidate IoTlistIOT ${ }_{i}$

For each $\mathrm{p}_{\mathrm{j}} \in \mathrm{IOT}_{\mathrm{i}}$, doCheck the application requirements' profiles

Communicate data from IOT to $\mathrm{CN}$.

STEP 9: CN respond back the query of user.

Return

\subsection{Proposed methodology}

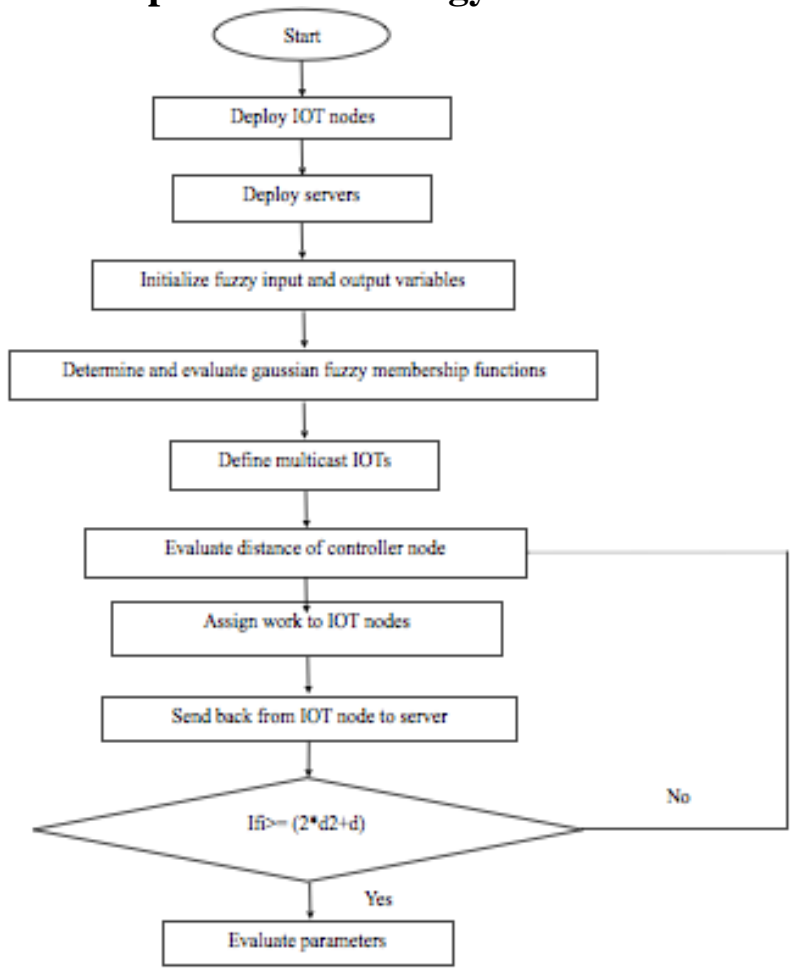

Fig 3: Proposed Methodology

\section{ANALYSIS OF RESULTS}

The proposed technique is designed and implemented in MATLAB tool u2013a. Here we will compare the performance of existing and proposed Fuzzy based IOTs by considering energy harvesting based sensor nodes. The graphical comparison has been carried out between proposed technique i.e. Gaussian functional shapes as membership functions of fuzzy logic with existing technique is triangular functions of fuzzy logic on the basis of parameters like consumption sleep mode, consumption transmission mode and throughput depends on the server i.e. no of IOTs is 11 with data packet size 1000 .

\subsection{Consumption Sleep Mode}

Consumption Sleep mode is a low power mode for various electronic devices such as air conditioners, televisions, computers, and other remote-controlled devices.

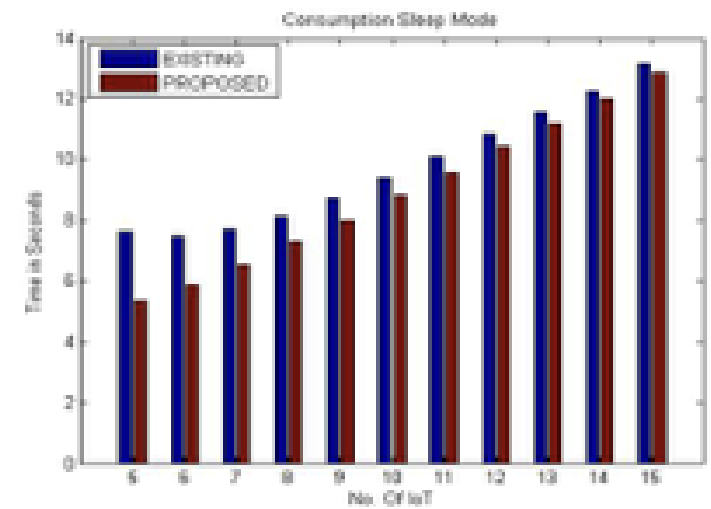

Fig 4: Performance Analysis of Consumption Sleep Mode 
The above figure 4 describes the comparison of consumption sleep mode between the existing and proposed technique where $\mathrm{x}$-axis shows the no of IOT and $\mathrm{y}$-axis represents consumption sleep time in seconds.

\subsection{Consumption Transmission Mode}

Consumption Transmission mode means transferring of data between two devices. And it tells that at what rate energy is consumed during transmission.

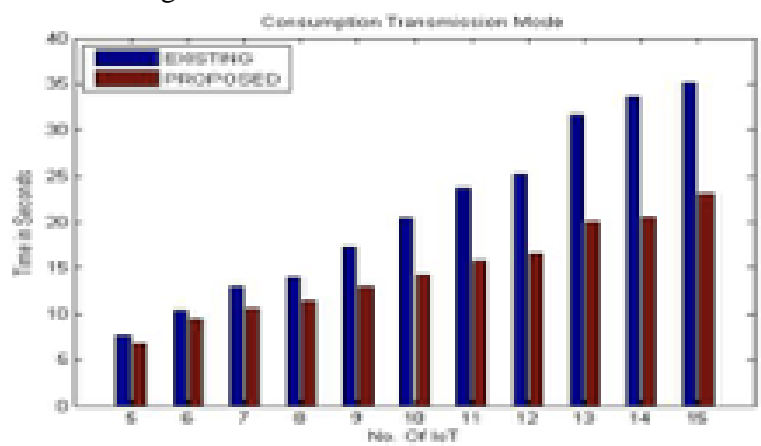

Fig 5: Performance Analysis of Consumption Transmission Mode

The above figure 5 describes the comparison of consumption transmission mode between the existing and proposed technique where $\mathrm{x}$-axis shows the no of IOT and y-axis represents time in seconds.

\subsection{Throughput}

It represents the number of communications executed per second, an application can manage, the amount of transactions made over time throughout a test.

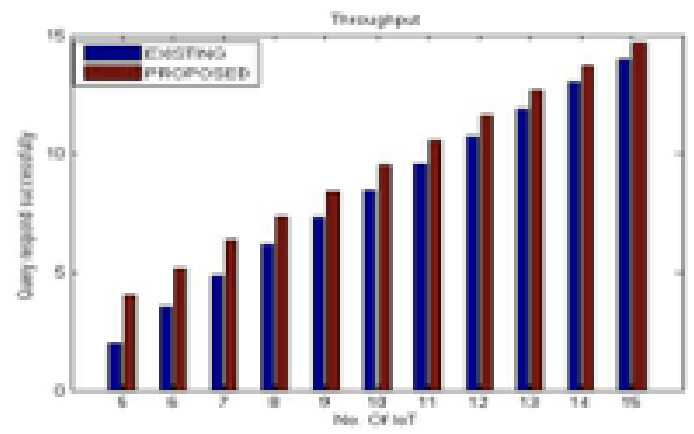

Fig 6: Performance of Throughput

The above figure 6 describes the comparison of throughput between the existing and proposed technique where $\mathrm{x}$-axis shows the no of IOT and $y$-axis represents the value.

\subsection{Execution Time}

The difference between the finish time of job and the start time of the job is called execution time. In table 2 shows that execution time using proposed algorithm decreases than existing technique.

$$
E_{x}=F_{t}-I_{t}
$$

Where $F_{t}$ finish is time and $I_{t}$ is Initial time

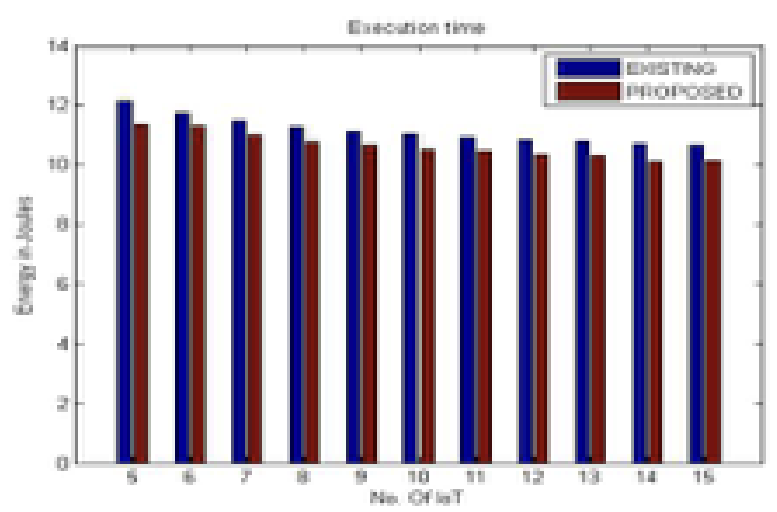

Figure 6: Performance Analysis of Execution time The above figure 6 represents the comparison of Execution time between the existing and proposed technique where $\mathrm{x}$ axis shows the no of iterations and y-axis shows the no of IOT.

\subsection{Energy Consumed}

Energy consumption is the amount of energy or power used. In table 3 represents that consumed Energy is comparatively lower than existing one.

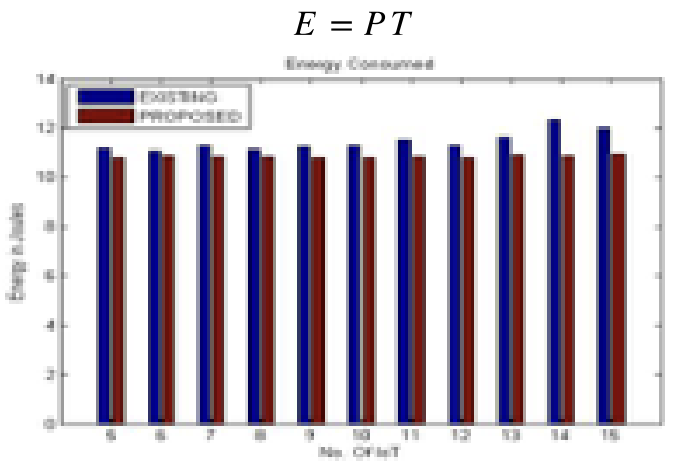

Figure 7: Performance Analysis of Energy Consumed

The above figure.7 describes the comparison of energy consumed between the existing and proposed technique where $\mathrm{x}$-axis shows the no of iterations and y-axis shows the no of IOT.

\subsection{Overhead}

Overhead is usually stated each and every combination of extra or indirect computation period, storage, data transfer rate or other assets that will are needed to acquire a particular objective. In table 4 shows that our proposed overheads are comparatively lower than the existing one.

Control Overhead $=$ Total Execution time - Computation time

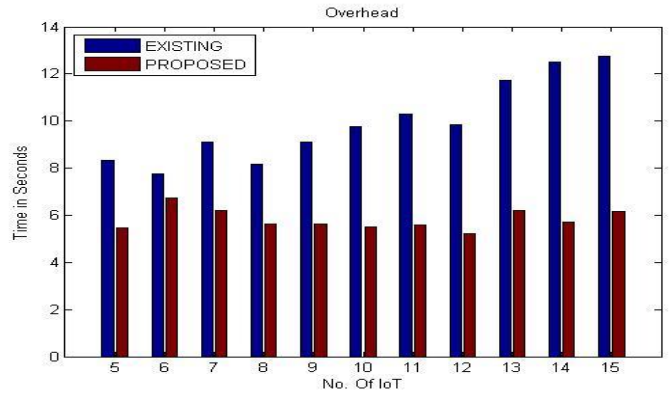

Figure 8: Performance Analysis of Overheads 
The above figure 8 represents the comparison of overheads between the existing and proposed technique where $\mathrm{x}$-axis describes the velocity and y-axis describes the no of IOT.

\section{REFERENCES}

[1] Romer, K., \&Mattern, F. (2004). The design space of wireless sensor networks. IEEE wireless communications, 11(6), 54-61.

[2] Hamel, M. J., Arms, S. W., Townsend, C. P., \& Churchill, D. L. (2006). U.S. Patent No. 7,081,693. Washington, DC: U.S. Patent and Trademark Office.

[3] Raghunathan, V., Ganeriwal, S., \& Srivastava, M. (2006). Emerging techniques for long lived wireless sensor networks. IEEE Communications Magazine, 44(4), 108-114.

[4] Du, X., Xiao, Y., Guizani, M., \& Chen, H. H. (2007). An effective key management scheme for heterogeneous sensor networks. Ad hoc networks, 5(1), 24-34.

[5] Akyildiz, I. F., Melodia, T., \& Chowdhury, K. R. (2008). Wireless multimedia sensor networks: Applications and testbeds. Proceedings of the IEEE, 96(10), 1588-1605.

[6] Gungor, V. C., \&Hancke, G. P. (2009). Industrial wireless sensor networks: Challenges, design principles, and technical approaches. IEEE Transactions on industrial electronics, 56(10), 4258-4265.

[7] Tripathy, M. R., Gaur, K., Sharma, S., \&Virdi, G. S. (2010, July). Energy efficient fuzzy logic based intelligent wireless sensor network. In Progress In Electromagnetics Research Symposium Proceedings, Cambridge, USA (pp. 91-95).

[8] Singh, S. K., Singh, M. P., \& Singh, D. K. (2010). A survey of energy-efficient hierarchical cluster-based routing in wireless sensor networks. International Journal of Advanced Networking and Application (IJANA), 2(02), 570-580.

[9] Kour, H., \& Sharma, A. K. (2010). Hybrid energy efficient distributed protocol for heterogeneous wireless sensor network. International Journal of Computer Applications, 4(6), 1-5.

[10] Ajofoyinbo, A. M., Olunloyo, V. O., \&Ibidapo-Obe, O. (2011). On development of fuzzy controller: The case of gaussian and triangular membership functions. Journal of Signal and Information Processing, 2(04), 257.

[11] Hameed, I. A. (2011). Using Gaussian membership functions for improving the reliability and robustness of students' evaluation systems. Expert systems with Applications, 38(6), 7135-7142.

[12] Mainetti, L., Patrono, L., \&Vilei, A. (2011, September). Evolution of wireless sensor networks towards the internet of things: A survey. In Software, Telecommunications and Computer Networks (SoftCOM), 2011 19th International Conference on (pp. 1-6). IEEE.

[13] Zarafshan, F., Karimi, A., \& Al-Haddad, S. A. R. (2012). A Novel Fuzzy Diffusion Approach for Improving Energy Efficiency in Wireless Sensor Networks.
International Journal of Machine Learning and Computing, 2(4), 506.

[14] Toulabi, M., \&Javadi, S. (2012). Energy-Saving in Wireless Sensor Networks Based on Optimization Sink Movement Control.

[15] Siew, Z. W., Wong, C. H., Kiring, A., Chin, R. K. Y., \&Teo, K. T. K. (2012). Fuzzy logic based energy efficient protocol in wireless sensor networks. ICTACT J. Commun. Technol.(IJCT), 3(4), 639-645.

[16] Chamodrakas, I., \&Martakos, D. (2012). A utility-based fuzzy TOPSIS method for energy efficient network selection in heterogeneous wireless networks. Applied Soft Computing, 12(7), 1929-1938.

[17] Lee, J. S., \& Cheng, W. L. (2012). Fuzzy-logic-based clustering approach for wireless sensor networks using energy predication. IEEE Sensors Journal, 12(9), 28912897.

[18] Basagni, S., Naderi, M. Y., Petrioli, C., \&Spenza, D. (2013). Wireless sensor networks with energy harvesting. Mobile Ad Hoc Networking: The Cutting-Edge Directions, 701-736.

[19] Gubbi, J., Buyya, R., Marusic, S., \&Palaniswami, M. (2013). Internet of Things (IoT): A vision, architectural elements, and future directions. Future generation computer systems, 29(7), 1645-1660.

[20] Andersson, M. (2014). Use case possibilities with Bluetooth low energy in IoT applications. White Paper.

[21] Borgia, E. (2014). The Internet of Things vision: Key features, applications and open issues. Computer Communications, 54, 1-31.

[22] Zanella, A., Bui, N., Castellani, A., Vangelista, L., \&Zorzi, M. (2014). Internet of things for smart cities. IEEE Internet of Things journal, 1(1), 22-32.

[23] Fang, W., Shan, L., Shi, Z., Jia, G., \& Wang, X. (2014). A Spatial Architecture Model of Internet of Things Based on Triangular Pyramid. In Mechatronics and Automatic Control Systems (pp. 825-832). Springer International Publishing.

[24] Vandikas, K., \&Tsiatsis, V. (2014, September). Performance Evaluation of an IoT platform. In Next Generation Mobile Apps, Services and Technologies (NGMAST), 2014 Eighth International Conference on (pp. 141-146). IEEE.

[25] Kim, S., Vyas, R., Bito, J., Niotaki, K., Collado, A., Georgiadis, A., \&Tentzeris, M. M. (2014). Ambient RF energy-harvesting technologies for self-sustainable standalone wireless sensor platforms. Proceedings of the IEEE, 102(11), 1649-1666.

[26] Ali, O. A. M., Ali, A. Y., \&Sumait, B. S. (2015). Comparison between the Effects of Different Types of Membership Functions on Fuzzy Logic Controller Performance. International Journal, 76.

[27] Kamalinejad, P., Mahapatra, C., Sheng, Z., Mirabbasi, S., Leung, V. C., \& Guan, Y. L. (2015). Wireless energy harvesting for the internet of things. IEEE Communications Magazine, 53(6), 102-108. 